- Instituto lécnico de la Construeción y del Cenento -

615-34 FABRICACION DE CEMENIO PORT LAND, DE FDURECIMIENTO BAPIDO

(The Use of Calcium Phosphate in Rapid-hardening Portland Cement).

I.D. Eratiov

De: "TSEMENT", $n=4,1955$ *

E1 cemento de endirecimiento răpido so define como aque?. cemento que alcanza en un dia el 50\%, como minimo, de su resistencia a los 28 dias, $y$ en tres dias, el $80 \%$. Aotualmente, ninguno de los cementos que se fabrican llegan a alcanzar, en un dia, más del $30 \%$ de su resistencia a los 28 dias.

El endurecimiento del cemento portland puedo acelorarse de diferentes formas. Una de ellas consiste on la adición de compues tos quimicos, capaces de elevar la roactividad de los principales com puestos del clínker, silicatos y aluminatos oélcicos. Los hidratos do estos dos oompuestos, asi como ol $\mathrm{Ca}(\mathrm{OH})_{2}$, constituyen los productios básicos finalos de la hidratación del cemento. Por consiguiente, la ve locidad de hidratación, la velocidad de crecimionto de la resisten cia, y otras propiedades del cemonto endurecido, son función do la actividad de estos compuestos, quedando afectadas por los cambios qui micos o físico-químioos que cxperimontan on sus propiedades durantc la hidrstactón.

Con el fin de ostudiar por separado la influencia do estos factores y determinar su acción sobre el desarrollo de resistoncia del comonto endurocido, se llevó a cabo una Investigación con cua tro tipos de cemento portland, cuyas composiciones quimicas y mineres lógicas quedan indicadas en la tabla $I$.

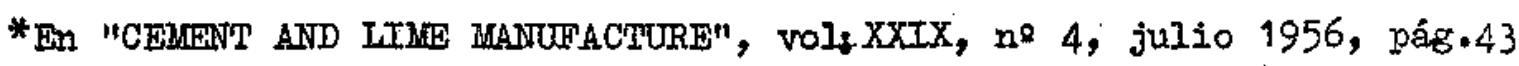


IABLA 1

\begin{tabular}{|c|c|c|c|c|c|c|c|c|}
\hline Cenento & $\mathrm{SiO}_{2}$ & $\begin{array}{c}\mathrm{Al}_{2} \mathrm{OB} \\
\%\end{array}$ & $\begin{array}{c}\mathrm{Fe}_{2} \mathrm{O}_{3} \\
\%\end{array}$ & $\%$ & ${ }^{3 \mathrm{CaO} . \mathrm{SiO}_{2}}$ & $\begin{array}{c}2 \mathrm{CaO}_{2} \mathrm{SiO}_{2} \\
\%\end{array}$ & 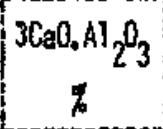 & $\begin{array}{c}4 \mathrm{CaO} . \mathrm{Al}_{2} \mathrm{O}_{3} \cdot \mathrm{Fe}_{2} \mathrm{O}_{3} \\
\%\end{array}$ \\
\hline $\begin{array}{l}\text { con alto contenido } \\
\text { on alita }\end{array}$ & 27.97 & 4.75 & 1.77 & 69.63 & 82.47 & 0.71 & 9.59 & 5.38 \\
\hline $\begin{array}{l}\text { con alto contenido } \\
\text { en belita }\end{array}$ & 25.30 & 6.90 & 4.60 & 63.20 & 9.61 & 62.25 & 10.47 & 13.80 \\
\hline Aluminoso & 22,30 & 6.85 & 1.95 & 68.00 & 50.32 & 19.20 & 15.60 & 5.85 \\
\hline $\begin{array}{l}\text { con alto contenido } \\
\text { en ferrito }\end{array}$ & 21.20 & 5.35 & 8.35 & 65.10 & 56.39 & 18.25 & - & 25.05 \\
\hline
\end{tabular}

En la tabla II se presenten los productos de hidrata ción de estos comentos, al cabo de 1, 3 y 28 dias. La resistencia mayor a los 28 días so consiguió con el cemento de alto contenido en alita, y la inferior con el de alto contenido en belita.

\section{IABLA II}

Composición (determinada por cálcuio) de los productos de hidratación de los cementos portland considerados

\begin{tabular}{|c|c|c|c|c|c|c|c|c|c|c|c|c|c|c|c|c|}
\hline \multirow{2}{*}{$\begin{array}{l}\text { Productos de } \\
\text { hidratación }\end{array}$} & \multicolumn{4}{|c|}{ cerento do alita } & \multicolumn{4}{|c|}{ cemento de belita } & \multicolumn{4}{|c|}{ cemento al umínoso } & \multicolumn{4}{|c|}{ comento de ferrito } \\
\hline & 1 & $\begin{array}{c}3 \\
\text { dfas }\end{array}$ & $\begin{array}{c}7 \\
\text { dias }\end{array}$ & total & $\begin{array}{c}1 \\
\text { dfa }\end{array}$ & $\begin{array}{c}3 \\
\text { dias }\end{array}$ & $\begin{array}{l}7 \\
\text { dras }\end{array}$ & total & $\begin{array}{c}1 \\
d f a\end{array}$ & $\begin{array}{c}3 \\
\text { dias }\end{array}$ & 7 & total & $\begin{array}{c}1 \\
d f a\end{array}$ & $\begin{array}{c}3 \\
\text { dias }\end{array}$ & d & total \\
\hline $\mathrm{Ca}(\mathrm{OH})_{2}$ & 6.0 & 12.0 & 20.0 & 25,0 & 0.5 & 1.0 & 20 & 3.0 & 4.0 & 8.0 & & 18.0 & 4.0 & 8.5 & 13,0 & 18.0 \\
\hline $2 \mathrm{C}=\mathrm{O}_{4} \mathrm{SiO}_{2} \mathrm{H}_{2} \mathrm{O}$ & 13.0 & $z, 0$ & 42.0 & 58.0 & 3.5 & 7. & 18.0 & 68.0 & 10.0 & 20.0 & & 56,0 & 8.0 & 17.0 & 33.0 & 56,0 \\
\hline $\mathrm{CCO}_{2} \mathrm{Al}_{2} \mathrm{O}_{3} \cdot \mathrm{OH}_{2} \mathrm{O}$ & 11.0 & 12.0 & 13.0 & 14.5 & 13.5 & 17.0 & 18.0 & 23.0 & 15.0 & 18,0 & & 23,0 & $B .0$ & 10.5 & 13.0 & 14.0 \\
\hline $\mathrm{C}_{2} \mathrm{O}_{0} \mathrm{~F}_{\mathrm{\theta}} \mathrm{O}_{3}, \mathrm{H}_{2} \mathrm{O}$ & 1.0 & 1.0 & 2.0 & 2.5 & 2.5 & 3.0 & 4.0 & 8.0 & 1.0 & 1.0 & 9.5 & 2.5 & 5.0 & 6.0 & 8.0 & 12.0 \\
\hline $\begin{array}{l}\text { clifnker sin desconim } \\
\text { porẹr. }\end{array}$ & 160,0 & 50.0 & 23.0 & - & 80.0 & 720 & 58.0 & - & 70.0 & 0 & 31.5 & - & 5.0 & 58.0 & 33.0 & .. \\
\hline
\end{tabular}


Estas dos tablas indican que, al principio de la hidratación, las centidades de hidróxido cálcico y de sllicato cálcico hi dratado son mucho mayores en el cemento de alto contenido en alita que en los restentes; pero, en cambio, el contenido en aluminatos hidratados y de $\mathrm{Fe}_{2} \mathrm{O}_{3}$ hidratado es menor en aquel tipo de cemento que en los restantes. Todo esto indica que las cantidades relativas de hidróxido cáloico y de silicato cálcico en los productos de hidratación pueden utilizarse como un criterio del desarrollo de la resis tencia al cabo de 1 y 3 dias.

Puesto que el silicato tricálcico puede considerarse como la principal fuente de hidróxido cálcico y de silicato cálcico en la fase inicial de la hidratación, el porcentaje de silicato tricálcico en el clinker, o, pera ser correcto, la relación de $\mathrm{C}_{3} \mathrm{~S}$ a $\mathrm{C}_{2} \mathrm{~S}$, se puede considerar como un factor importante on el desarrollo de re sistencia a edades superiores. Esta relación se defino como "módulo de intensidad". "Cuanto mayor es su valor, tanto meyor seré la resistencia del comento a edades superiores. Con el fin de poner de maniflesto quo esta suposición era correcta; se investigaron diez clínly res diferontes, (tabla III) con mödulos do intensidad quo variaban.. desde 3 a 20. Ia tabla IV pone de manifiesto que, a 24 horas, un aumento del módulo de intensidad de 3 a 20 determina un aumento do la resistencia a compresión de $180 \ldots .400 \mathrm{Kg} / \mathrm{cm}^{2}$. Es, pues, evidonte que interesa un módulo de intensidad lo mayor posibilo. (Vex pági na siguiente).

Teniendo on euanta las dificultades con que se tropieza para fabricar un clinker con un contenido elevado en alita, se añe dieron productos quimicos capaces de modificar la velocidad de hiare tación. Las investigaciones realizadas pusieron de manifiesto que, - 
TA BLA III

Composición mineralógica de los cl lnkeros ensayados

\begin{tabular}{|c|c|c|c|c|c|c|c|}
\hline \multirow{2}{*}{ Cinker } & \multicolumn{3}{|c|}{ Relaciones (\%) } & \multicolumn{4}{|c|}{ Composición calculada } \\
\hline & $\frac{c_{3} s}{C_{2} s}$ & $\frac{C_{3} A}{C_{4} A F}$ & $\frac{C_{2} S+C_{3} S}{C_{3} A+C_{4} A F}$ & $C_{3} S$ & $\mathrm{C}_{2} \mathrm{~S}$ & $C_{3} A$ & $\mathrm{C}_{4} \mathrm{AF}$ \\
\hline 1 & 3 & 0.33 & 4 & 60.0 & 20.0 & 5.00 & 15.00 \\
\hline 2 & 3 & 3.00 & 4 & 60,0 & 20.0 & 15.00 & 5.00 \\
\hline 3 & 5 & 0.33 & 4 & 66.7 & $\$ 3.3$ & 5,00 & 15.00 \\
\hline 4 & 5 & $3.00^{\circ}$ & 4 & 66.7 & 13.3 & 15.00 & 5.00 \\
\hline 5 & 10 & 0.33 & 3 & 68.2 & 6.8 & 6.25 & 18.75 \\
\hline 6 & 10 & 3.00 & 3 & 88.2 & 6.8 & 18.75 & 6.25 \\
\hline 7 & 45 & 0,33 & 3 & 70,3 & 4.7 & 6.25 & 18.75 \\
\hline 8 & 15 & 3.00 & 3 & 70.3 & 4.7 & 18.75 & 6.25 \\
\hline 9 & 20 & 0.33 & 5 & 79.4 & 4.0 & 4.2 & 12.40 \\
\hline 10 & 20 & 3.00 & 5 & 79.4 & 4.0 & 124 & 4.2 \\
\hline
\end{tabular}

TABLA IV

Resistencia de los cl lnkeres sintéticos

\begin{tabular}{|c|c|c|c|c|c|c|c|c|}
\hline \multirow{2}{*}{$\begin{array}{c}\text { Clfthker } \\
n^{0}\end{array}$} & \multicolumn{4}{|c|}{ Assistencla a comprasion $(\mathrm{kg} / \mathrm{cm})^{2}$} & \multicolumn{4}{|c|}{ Resistencia a tracción ( $\left.\mathrm{kg} / \mathrm{c}^{2}\right)$} \\
\hline & $\partial \gamma_{a}$ & diss & d & dics & día & dlas & dlas & dias \\
\hline 1 & 180 & 290 & 514 & 605 & 23,9 & 2.4 & 26.9 & 32.1 \\
\hline 2 & 225 & 3,36 & 506 & 619 & 25.1 & 27.2 & 29.8 & 33.7 \\
\hline 3 & 237 & 391 & 509 & 625 & 24.9 & 28.6 & 28.9 & 32.2 \\
\hline 4 & 260 & 406 & 502 & 640 & 20.9 & 28.7 & 29.3 & 34.1 \\
\hline 5 & 280 & 42 & 601 & 653 & 200.7 & 29,0 & 31.8 & 36,1 \\
\hline 6 & 334 & 482 & 649 & 771 & 20.7 & 3.2 .1 & 38.2 & 44.1 \\
\hline 7 & $3 x$ & 435 & 602 & $7 r_{1} 1$ & 28.8 & 31.7 & 36.4 & 40.6 \\
\hline 8 & 395 & 524 & 673. & 815 & 3.3 .1 & 35.6 & 40.5 & $4 \pi, 5$ \\
\hline 9 & 350 & 490 & 642 & 743 & 30.5 & 36.3 & 35.1 & 40.2 \\
\hline 10 & 400 & 536 & 571. & 205 & 20.1 & 34,6 & 33.9 & 42. 1. \\
\hline
\end{tabular}


añadiendo $\mathrm{Ca}_{3}\left(\mathrm{PO}_{4}\right)_{2}$ a la pasta cruda, el clinker resultante conte nia un contenido elevado en fosfato oálcico que, como tal o en solu ción sólida con el silicato tricálcico, podian modificar intensamen te el fraguado del cemento.

In la tabla- $V$ se presentan los datos concernientes a la influenoia del contenido en $\mathrm{P}_{2} \mathrm{O}_{5}$.

\section{IABLA}

Efecto del $\mathrm{P}_{2} \mathrm{O}_{5}$ sobre la resistencia del cemento.

\begin{tabular}{|c|c|c|c|c|}
\hline \multirow{2}{*}{$\begin{array}{l}\text { Contenido en } \\
\text { Pas on al anto } \\
\text { comento }\end{array}$} & \multicolumn{4}{|c|}{$\begin{array}{c}\text { Resistencia a compresion } \\
\left(\mathrm{kg} / \mathrm{cm}^{2}\right)\end{array}$} \\
\hline & $d a$ & días & dias & dias \\
\hline 0.0 & 118 & $266^{\circ}$ & 360 & 535 \\
\hline 0.1. & 258 & 370 & $48^{4}$ & 685 \\
\hline 0.2 & 352 & 463 & 560 & 745 \\
\hline 0.3 & 319 & 388 & 495 & 655 \\
\hline 0.5 & 201 & 269 & 374 & 538 \\
\hline 1.0 & $\zeta$ & 106 & 202 & 388 \\
\hline 20 & 52 & 84 & 150 & 274 \\
\hline 3.0 & 35 & 73 & 107 & 105 \\
\hline 5.0 & 0 & 27 & 58 & 72 \\
\hline
\end{tabular}

Se observó que una pequeña cantidad $(0,2 \ldots \ldots ., 3 \%)$ de $\mathrm{P}_{2} \mathrm{O}_{5}$ en el clinker tenja un efecto extreordinariamente crande sobre la hidratación y desarrollo de la resistencia, no sólo injoial sino también a los 28 dias. 